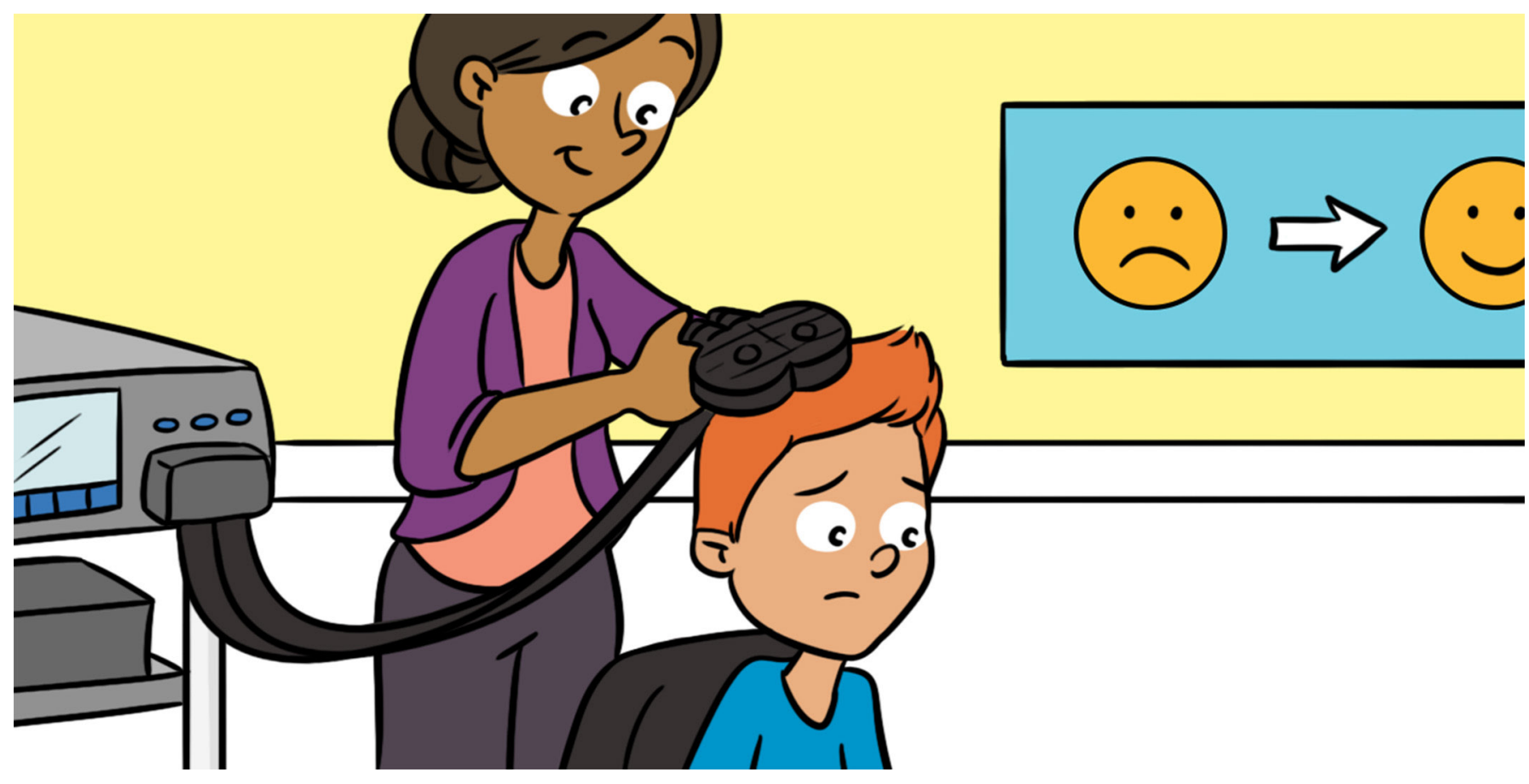

\title{
USING MAGNETS TO STIMULATE THE BRAIN HELPS PEOPLE WITH DEPRESSION
}

\section{Manreena Kaur ${ }^{1,2 *}$, Karyn E. Richardson ${ }^{1,2}$ and Paul B. Fitzgerald ${ }^{1,2}$}

${ }^{1}$ Monash Alfred Psychiatry Research Centre, Monash University Central Clinical School and the Alfred, Melbourne, VIC, Australia ${ }^{2}$ Epworth Centre for Innovation in Mental Health, Epworth Healthcare, Melbourne, VIC, Australia

\section{YOUNG REVIEWERS:}

\begin{tabular}{l}
\hline \\
MATTHS \\
FLINDERS \\
Anglican college
\end{tabular}

MATTHEW FLINDERS ANGLICAN COLLEGE

AGES: $14-15$
Depression is a common and disabling mental illness that can be difficult to treat and lasts a long time. Typical treatment options for depression are antidepressant medications and psychological therapies, which are helpful for some people but do not work for everyone. The underlying cause of depression is not well-understood, but one key problem is that brain cells do not communicate with each other as well as they should. Transcranial magnetic stimulation is a technique that can help to improve the communication between brain cells and this technique improves depression in many people who do not get better with antidepressant medication. However, this treatment is often not available to many patients with depression who may benefit from it, because it is expensive and takes up a lot of the patients' time. Researchers are now working toward making this treatment more accessible, so that many more people with depression can benefit from it. 


\section{NEUROTRANSMITTER}

A group of chemical messengers that are exchanged between neurons as they communicate.

\section{ACTION POTENTIAL}

The electric impulse that travels through a neuron, changing the electric charge of the neuron.

\section{SYNAPSE}

The gap in between two neurons where neurotransmitters are exchanged in communication between the two neurons.

\section{Figure 1}

(A) When a TMS pulse is applied to the brain, action potentials are generated in the neurons under the skull. (B) When an action potential happens, the electrically charged neuron becomes more positively charged, a process called depolarization. (C) When the action potential travels through the neuron, the neuron releases neurotransmitters into the synapse, the space in between neurons. The neurotransmitters are then taken up by the second neuron, triggering an action potential in that neuron.

\section{BRAIN CELLS NEED TO COMMUNICATE WITH EACH OTHER}

The human brain is made up of billions of cells called neurons. There are an average of 100 billion neurons in our brains [1]! Are you wondering how all those neurons fit inside our heads? It is because they are very small ( $<0.5 \mathrm{~mm}$ in diameter) and packed together very tightly. There are many different types of neurons and they all have different jobs. To do their jobs well, they have to work as a team and communicate with each other. It is like doing a group assignment in school, in which all the students on a team are assigned different tasks to complete the project. If one person does not do her/his part, then it will impact the rest of the team! Neurons communicate with each other through "chemical signaling," a process in which they exchange chemicals called "neurotransmitters." This is how it happens: an "action potential" is an electrical impulse that travels through a neuron, triggering it to release neurotransmitters into a small gap found in between two neurons, called a "synapse." Then, the neuron on the other side of the synapse takes up the neurotransmitters, which then generates an action potential that travels through this neuron. This process continues in several neurons that are connected to each other. Have a look at Figure $1 \mathrm{C}$ to see how neurons communicate with each other. Our abilities to pay attention to things in the environment, to remember what we learn and experience, to make decisions every day, and to manage our emotions all depend on how well our neurons work together as a team. When neurons cannot do their jobs properly, there is a domino effect on other neurons and because of this, important brain functions cannot be carried out properly. This is what happens to the brain when people experience a mental illness.

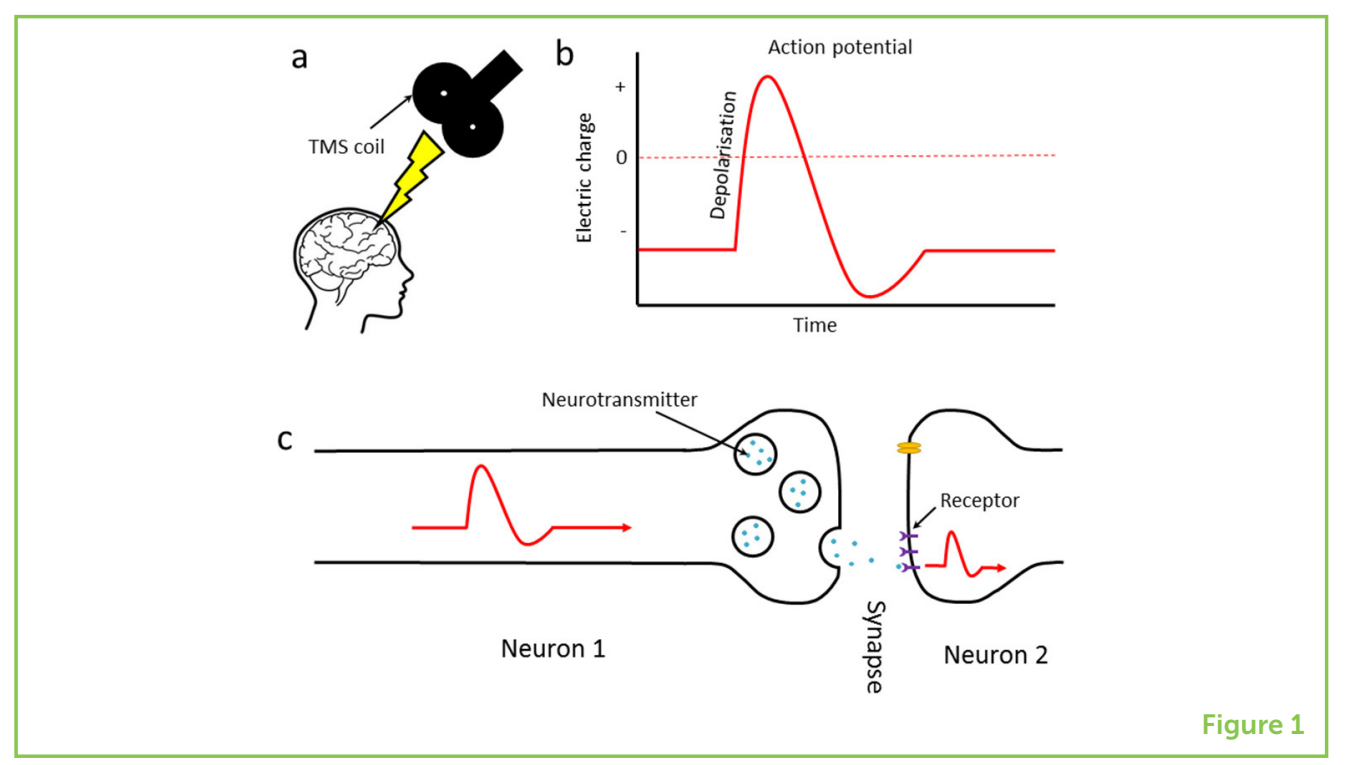




\section{WHAT IS IT LIKE TO HAVE DEPRESSION?}

Depression is the most common mental illness [2]. $4.4 \%$ of the world's population suffers from depression. This is an alarming 322 million people, which is about the total population of the U.S.A! For most people, depression starts when they are young, before 30 years of age, and as depression often lasts a long time, many people suffer from it for a large part of their lives [3]. People with depression experience prolonged periods of feeling very sad and/or lose the ability to feel pleasure. They commonly describe a feeling of emptiness, worthlessness, guilt, loss of hope, or lack of interest in many activities they used to enjoy. These feelings are often accompanied by other symptoms, such as trouble thinking clearly or carrying out day-to-day tasks, difficulty sleeping at night, feeling fatigued during the day, and reduced appetite. Depression is the leading cause of disability world-wide and many people who suffer from it cannot function well-enough to work, study, or maintain their relationships with family and friends.

\section{HOW CAN WE IMPROVE COMMUNICATION BETWEEN NEURONS TO HELP PEOPLE WITH DEPRESSION?}

Scientists do not fully understand all the complicated brain changes that cause depression. We know that there are several factors that play a role. Two of these factors are the abnormal activity of many different types of neurotransmitters and reduced ability of neurons to fix themselves when they malfunction [4]. Together, these factors result in poor communication between neurons in many regions of the brain.

Antidepressant medication can help people with depression by improving communication between neurons. While antidepressants work for many people with depression, at least half of patients are not able to achieve or sustain full recovery from their depression with antidepressants [3]. Another problem with antidepressants is that they cause undesirable side effects, such as weight gain, which can lead to other health problems. These side effects occur because medications that are swallowed are absorbed through the gut, and therefore affect cells throughout the body, not just in the brain. Psychological therapy (also called talk therapy) is also widely used to treat depression. It involves the patients seeing psychiatrists or psychologists who guide them to learn how to think about their feelings of depression differently. By training yourself to change the way you think about your feelings, you are organizing the neurons in certain regions of the brain so that they get better at communicating with each other [4]. The problem with psychological therapy is that it also does not work for all patients and 
it can take many months or even years before depression symptoms are treated. So, the treatments we typically use for depression have limitations and it is very important for researchers to find new, more effective treatments.

\section{USING A MAGNETIC FIELD TO CHANGE THE ACTIVITY OF NEURONS}

As described in 1831 by the famous physicist Michael Faraday, a brief magnetic field applied to a material that conducts electricity will generate an electrical current flow [5]. Do you remember that an action potential is an electrical impulse that travels through a neuron? This means neurons are electrically charged and can conduct electricity! So, applying a magnetic field will cause current to flow through the neurons and this can alter their activity. To alter the activity of neurons, the electric field must be strong enough to generate an electrical current in the neurons as well as in the synapses in between them [1]. When the electric charge is strong enough, the neuron will become "depolarized," meaning that it becomes more positively electrically charged. Have a look at Figure 1B showing the change in electric charge over time during an action potential. When a neuron is depolarized, an action potential is produced. When the action potential reaches the end of the neuron, it triggers the release of neurotransmitters. The neurotransmitters then attach to "receptors" on the next neuron across the synapse, and these receptors take up the neurotransmitters into the receiving neuron. This

\section{DEPOLARIZATION}

When a neuron's electrical charge becomes more positive, which occurs during an action potential.

\section{TMS}

Transcranial magnetic stimulation; a technique in which a magnetic pulse is applied to the scalp and travels through the skull to the brain, where it induces an electric current in neurons. then triggers depolarization in this neuron and then, an action potential. Figure 1 illustrates these processes. Now, can you imagine how applying current flow to neurons on the surface of the brain can set off a domino effect of communication to all the other neurons that are connected to it through synapses?

\section{STIMULATING THE BRAIN WITH MAGNETS CAN IMPROVE DEPRESSION}

In 1985, a device was invented that could alter the activity of neurons by applying a magnetic field on the scalp, a technique called TMS, which stands for transcranial magnetic stimulation [5]. Transcranial means that the magnetic field goes through the cranium (or skull) and magnetic stimulation refers to the use of a magnetic field to stimulate the neurons beneath the cranium. When TMS was first invented, researchers were very excited to use it to figure out which parts of the brain were connected to the different parts of the body. For example, they learnt that applying TMS to a certain part of the scalp made a person's hand twitch! This is how the part of the brain responsible for controlling hand 
Figure 2

Diagram of the electrical activity generated in the brain when TMS is applied to the left pre-frontal cortex. Each picture shows the brain from different angles. Cooler colors indicate increasing negative electric charge and warmer colors indicate increasing positive electric charge. These pictures clearly show how much of the brain is stimulated by TMS applied to the scalp.

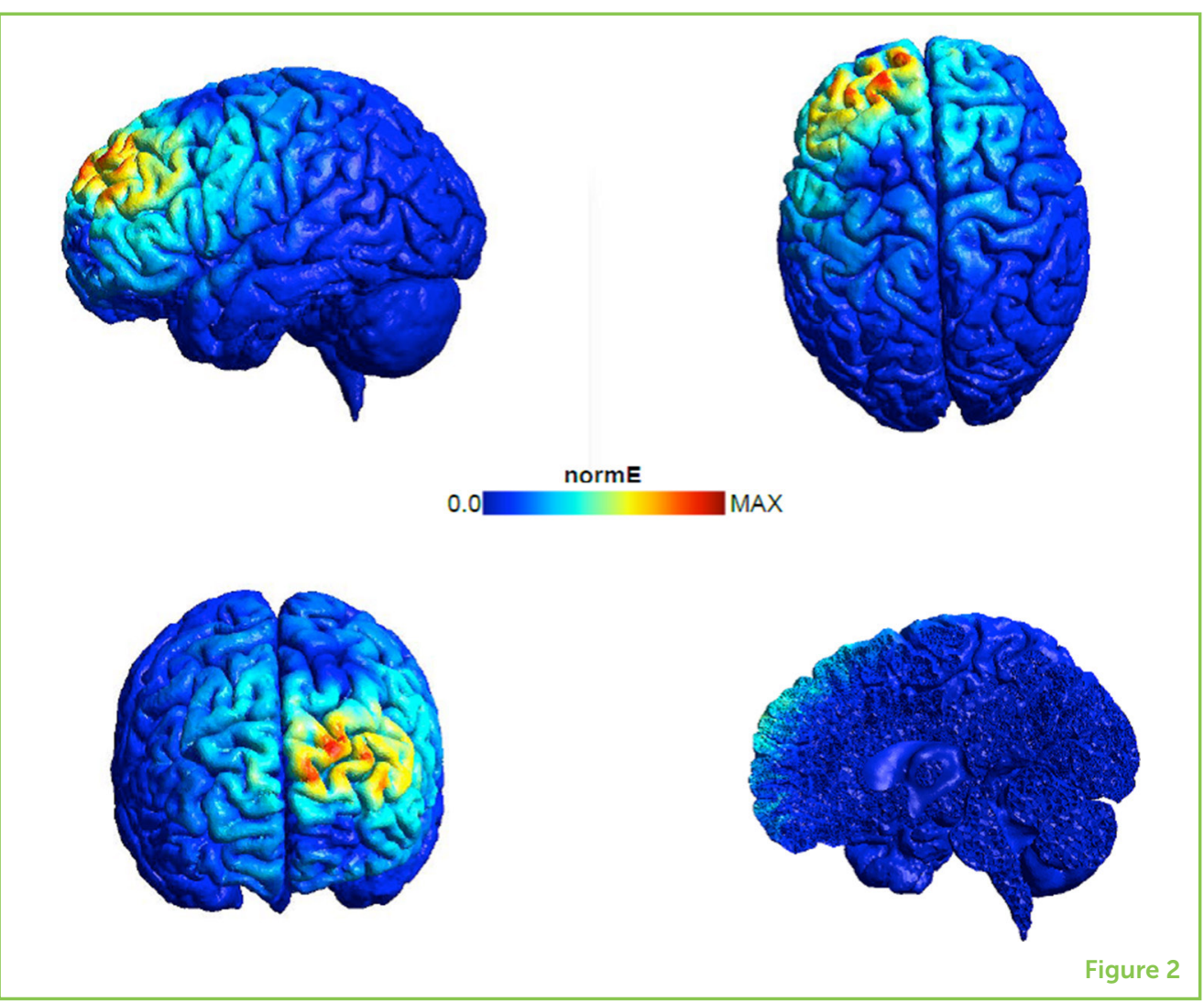

muscle movements was discovered! Interest in whether TMS could treat depression grew in the 1990's, when the first research studies using brain scanning to look at pictures of the brain in depression were carried out. These studies showed that the left side of the pre-frontal cortex, a region of the brain, was underactive in depression, leading researchers to question whether they could use TMS to increase the activity of neurons in this brain region. The initial findings of TMS applied to the left pre-frontal cortex for depression found that depression symptoms improved, which was very exciting! Have a look at Figure 2 to see the pattern of electrical activity when TMS is applied on the pre-frontal cortex of the brain.

There has been a tremendous amount of research on TMS treatment for depression in the past 20 years. Typically, TMS treatment consists of 20 min of treatment, 5 days a week, for 4 weeks. In Figure 3, you can see a photo of a patient receiving TMS. TMS is quite safe. The common side effects are mild, such as a mild headache, and severe side effects are very rare. As you can see in Figure 4, compared with antidepressant medication, there are many fewer side effects seen with TMS, and these side effects occur only around the scalp and not throughout the body. Several very careful studies performed on large numbers of patients have shown that TMS applied to the left pre-frontal cortex is effective for treating depression in people who do not improve with antidepressants. TMS has even been shown to improve the thinking skills that 


\section{Figure 3}

A patient receiving transcranial magnetic stimulation.

\section{Figure 4}

Comparison of side effects people experience with antidepressant medication versus TMS treatment. You can see that TMS treatment results in fewer side effects than antidepressant medication.

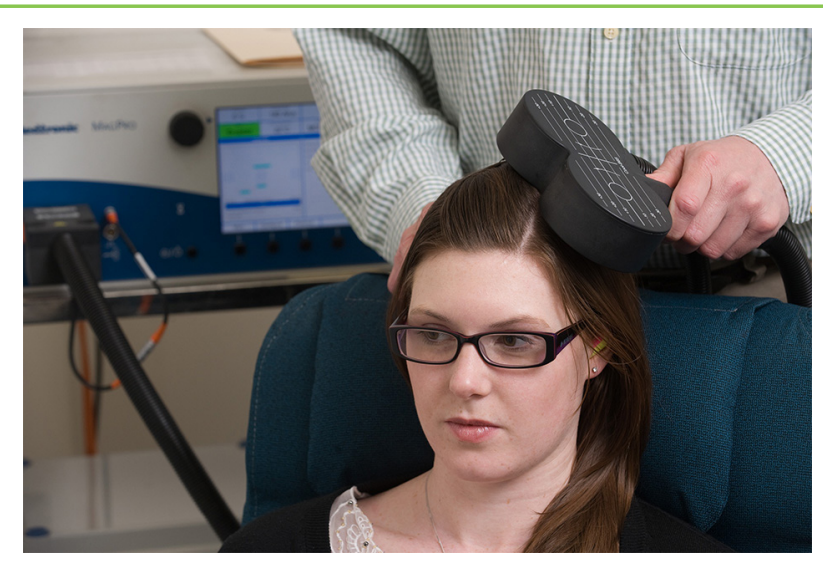

Figure 3

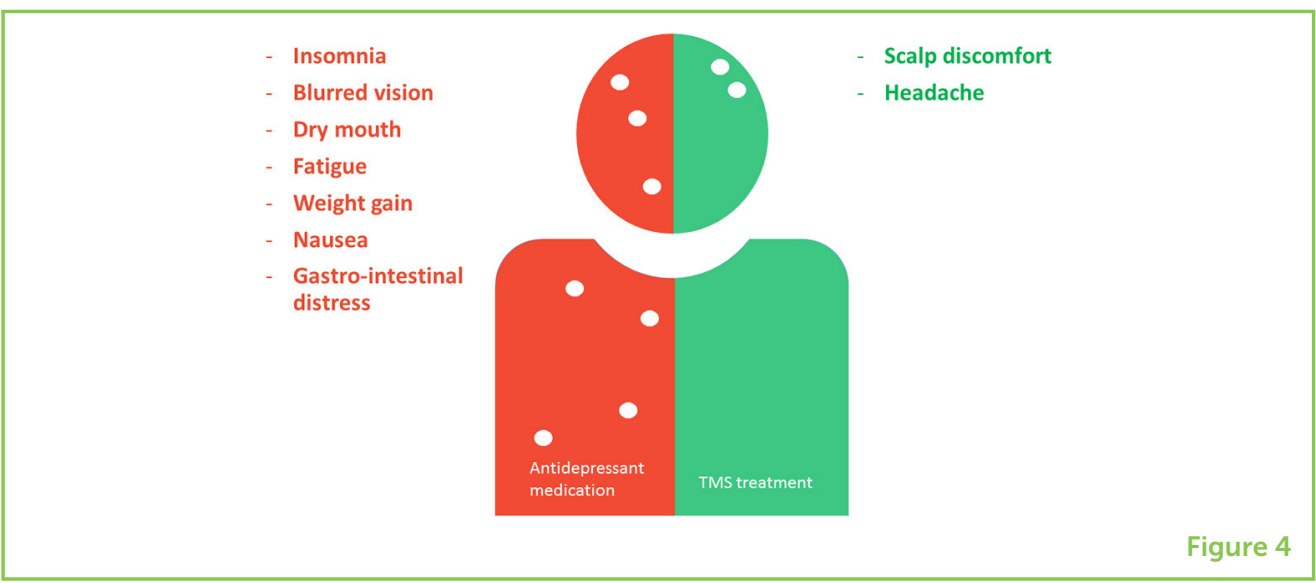

are normally affected in depression. These positive results have led to the approval of TMS as a treatment for depression by several governing bodies world-wide. However, like other depression treatments, TMS does not work for everyone-it works for about half the patients for whom medication does not work. Every year, TMS gives patients with depression new hope and changes thousands of lives. Here is an example of what one of our patients who received TMS for depression at the Monash Alfred Psychiatry Research Centre (Australia) said: "I had been in and out of hospital for months and tried every antidepressant ever made ... then my psychiatrist suggested TMS, and after the first session I already felt much better. For the first time in years, I could think clearly and make rational decisions. I felt on top of the world, like it saved my life."

\section{THE FUTURE OF TMS TREATMENT FOR DEPRESSION}

Since TMS has to be performed by medical professionals and it takes a lot of time, TMS is expensive and not all patients can afford it. Our goal now is to make TMS more accessible, so that many more patients can 
benefit from it. We are helping to make TMS accessible by researching ways to shorten the TMS treatment course and lobbying for some of the costs to be covered by health insurance or government-provided healthcare. Offering hope to patients with depression, particularly those who do not have other treatment options, makes the area of TMS research incredibly important and valuable.

\section{ACKNOWLEDGMENTS}

We would like to thank Aron T. Hill for creating Figure 3, the electrical field potential of transcranial magnetic stimulation on the left prefrontal cortex.

\section{REFERENCES}

1. Wickens, A. 2009. Introduction to Biopsychology. Harlow: Pearson Education.

2. World Health Organization. 2017. Depression and Other Common Mental Disorders: Global Health Estimates. Geneva: World Health Organization.

3. McIntyre, R. S., Filteau, M. J., Martin, L., Patry, S., Carvalho, A., Cha, D. S., et al. 2014. Treatment-resistant depression: definitions, review of the evidence, and algorithmic approach. J. Affect. Disord. 156:1-7. doi: 10.1016/j.jad.2013.10.043

4. Dean, J., and Keshavan, M. 2017. The neurobiology of depression: an integrated view. Asian J. Psychiatr. 27:101-11. doi: 10.1016/j.ajp.2017.01.025

5. Fitzgerald, P. B., and Daskalakis, Z. J. 2013. Repetitive Transcranial Magnetic Stimulation Treatment for Depressive Disorders: A Practical Guide. Berlin; Heidelberg: Springer Science \& Business Media.

SUBMITTED: 19 September 2018; ACCEPTED: 05 February 2019; PUBLISHED ONLINE: 20 February 2019.

EDITED BY: Daniel F. Hermens, Sunshine Coast Mind and Neuroscience Thompson Institute, University of the Sunshine Coast, Australia

CITATION: Kaur M, Richardson KE and Fitzgerald PB (2019) Using Magnets to Stimulate the Brain Helps People With Depression. Front. Young Minds 7:26. doi: 10.3389/frym.2019.00026

CONFLICT OF INTEREST STATEMENT: In the last 3 years, PF has received equipment for research from Magventure A/S, Medtronic Ltd, Neurosoft and Brainsway Ltd. He has served on a scientific advisory board for Bionomics Ltd and LivaNova and is a founder and board member of TMS Australia. The remaining authors declare that the research was conducted in the absence of any commercial or financial relationships that could be construed as a potential conflict of interest. 

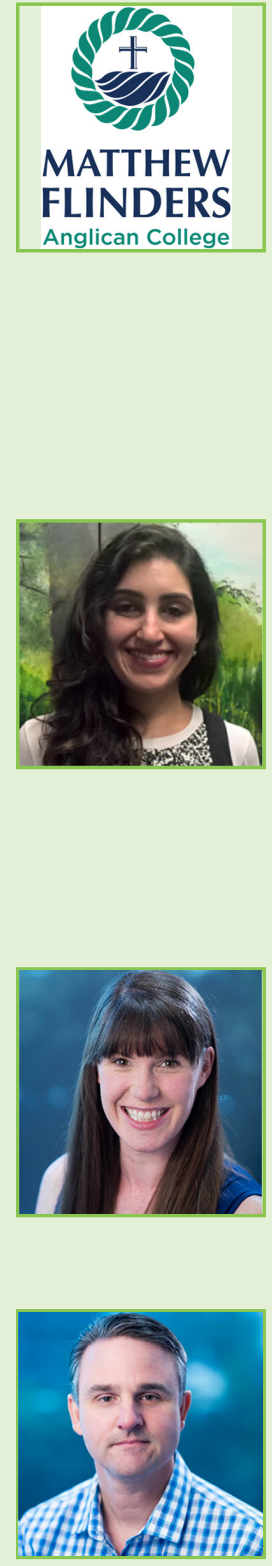

COPYRIGHT () 2019 Kaur, Richardson and Fitzgerald. This is an open-access article distributed under the terms of the Creative Commons Attribution License (CC BY). The use, distribution or reproduction in other forums is permitted, provided the original author(s) and the copyright owner(s) are credited and that the original publication in this journal is cited, in accordance with accepted academic practice. No use, distribution or reproduction is permitted which does not comply with these terms.

\section{YOUNG REVIEWERS}

\section{MATTHEW FLINDERS ANGLICAN COLLEGE, AGES: 14-15}

Matthew Flinders Anglican College young reviewers are keen budding scientists who enjoy taking on challenges. They are a collaborative bunch of young minds, with shared interests in science, music, and sport. This group of young reviewers thoroughly enjoy being part of Frontiers for Young Minds and are excited to be involved in how science is being communicated and shaped.

\section{AUTHORS}

\section{MANREENA KAUR}

As a Research Fellow at the Monash Alfred Psychiatry Research Centre, the focus of my research is on investigating treatments using brain stimulation techniques for depression and schizophrenia, particularly for young people. I have always been fascinated by how the body, brain, and mind work and I am dedicated to improving the lives of people with mental illness by helping to provide new treatment options for them. When I am not doing Science, I love to listen and explore new music, travel around the world, and hang out with my friends and family! *manreena.kaur@monash.edu

\section{KARYN E. RICHARDSON}

I am a Researcher and Psychologist with a passion for understanding how the brain works and influences the way we behave. I have worked on a number of studies using brain stimulation to treat depression and am interested in learning how we could use brain stimulation to help people who have experienced a stroke. In my free time, I enjoy spending time with family in the outdoors.

\section{PAUL B. FITZGERALD}

I am a Professor of Psychiatry at the Epworth Clinic and Monash Alfred Psychiatry Research Centre. Through my work as a Psychiatrist, I saw a strong need for more effective treatments for people with mental illness. I have since dedicated my career to understanding how the brain works and developing new brain stimulation treatments for people with mental illness. I also work to make these treatments easily accessible for patients. My interest in magnetic and electrical brain stimulation treatments may or may not have anything to do with have been struck by lightning when I was in my early 20 s. 